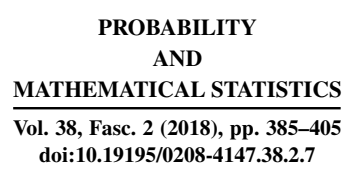

\title{
SUPERMODULAR ORDERING OF POISSON AND BINOMIAL RANDOM VECTORS BY TREE-BASED CORRELATIONS*
}

BY

\author{
BÜNYAMIN KIZILDEMIR (SINGAPORE) AND NICOLAS PRIVAULT (SINGAPORE)
}

\begin{abstract}
We construct a dependence structure for binomial, Poisson and Gaussian random vectors, based on partially ordered binary trees and sums of independent random variables. Using this construction, we characterize the supermodular ordering of such random vectors via the componentwise ordering of their covariance matrices. For this, we apply Möbius inversion techniques on partially ordered trees, which allow us to connect the Lévy measures of Poisson random vectors on the discrete $d$-dimensional hypercube to their covariance matrices.
\end{abstract}

2010 AMS Mathematics Subject Classification: Primary: 60E15; Secondary: 62H20, 05C05, 06A11, 60E07.

Key words and phrases: Stochastic ordering, supermodular functions, Möbius transform, Möbius inversion, binary trees, Poisson random vectors, binomial random vectors.

\section{INTRODUCTION}

A $d$-dimensional random vector $X=\left(X_{1}, \ldots, X_{d}\right)$ is said to be dominated by another random vector $Y=\left(Y_{1}, \ldots, Y_{d}\right)$ in the supermodular order, and one writes $X \leqslant \mathrm{sm} Y$, if

$$
E[\Phi(X)] \leqslant E[\Phi(Y)]
$$

for all integrable supermodular functions, i.e., for all functions $\Phi: \mathbb{R}^{d} \rightarrow \mathbb{R}$ such that

$$
\Phi(x)+\Phi(y) \leqslant \Phi(x \wedge y)+\Phi(x \vee y), \quad x, y \in \mathbb{R}^{d},
$$

where the maximum $\vee$ and the minimum $\wedge$ are defined with respect to the componentwise order of $x, y \in \mathbb{R}^{d}$. The supermodular stochastic ordering is used in particular to capture a preference for greater interdependence in economic variables. In other words, we have $X \leqslant_{\mathrm{sm}} Y$ if the (positive) dependence among the

* This research was supported by Singapore MOE Tier 1 Grant MOE2015-T1-2-130 RG122/15. We thank two anonymous referees for useful suggestions. 
components of $Y$ is greater than the (positive) dependence among the components of $X$. See, for example, [2] and [3] for applications of supermodular ordering in insurance, and [9] for applications to portfolio risk management, cf. also [8] and references therein for applications in economics.

In the case where $X$ and $Y$ are multivariate Gaussian vectors, the supermodular ordering of $X$ and $Y$ has been characterized by the componentwise ordering of their covariance matrices in [10]. Sufficient conditions for the supermodular ordering of general random vectors have been given in [4] for general random vectors, including Poisson and gamma vectors, cf. Section 4.2 therein. We note that our recursive update of Bernoulli random vectors in (5.8) below consists in an implementation on binary trees of the formulas in Section 4.2 of [4] for Poisson and gamma vectors.

In this paper, we construct a tree-based covariance structure for binomial and Poisson random vectors, under which the supermodular ordering can be characterized by the ordering of covariance matrices, cf. Theorems 4.$]$ and 5.1 . This approach uses Möbius inversion techniques which allow us to connect partially ordered binary trees on the discrete unit hypercube $\{0,1\}^{d}$ to supermodular ordering. We also show the necessity of dependence structure of this type in Counterexample 4.1. Other types of tree-based dependence structures in the setting of Bernoulli random vectors have been developed in [5] and references therein.

We proceed as follows. In Section $\square$ we construct a general dependence structure based on independent variables arranged according to a binary tree on the vertices of the $d$-dimensional hypercube. In Section B we describe the Möbius inversion that allows one to recover the parameters of individual random variables from the covariance matrix of the considered random vector. In Section 4 we deal with the case of Poisson random vectors via the use of Lévy measures on the vertices of the discrete unit hypercube $\{0,1\}^{d}$, cf. Theorem 4.1 . In Section $[5$ we apply this dependence structure to the characterization of the binomial supermodular ordering via the componentwise ordering of covariances, $\mathrm{cf}$. Theorem 5 .1]. This result naturally extends to the supermodular ordering of sums of binomial, multivariate Gaussian and Poisson random vectors.

\section{TREE-BASED CORRELATION STRUCTURES}

In this section we introduce the general dependence structure used in this paper. Let $\left(e_{1}, \ldots, e_{d}\right)$ denote the canonical basis of $\mathbb{R}^{d}$, and let

$$
C_{d}:=\{0,1\}^{d}=\left\{x=\left(x_{1}, \ldots, x_{d}\right): x_{i} \in\{0,1\}, i=1, \ldots, d\right\}
$$

denote the discrete set of vertices of the $d$-dimensional unit hypercube.

Every $x=\left(x_{1}, \ldots, x_{d}\right) \in\{0,1\}^{d}$ is identified with its index set

$$
S_{x}:=\left\{i \in\{1, \ldots, d\}: x_{i}=1\right\},
$$


and we endow $C_{d}=\{0,1\}^{d}$ with the partial inclusion ordering of index sets, i.e., we write

$$
x \preceq y \quad \text { when } 0 \leqslant x_{i} \leqslant y_{i} \leqslant 1, i=1, \ldots, d,
$$

and $x \prec y$ when $x \preceq y$ and $x \neq y$; we also let $x \backslash\{a\}$ denote $\left(x_{i} \mathbf{1}_{\{i \neq a\}}\right)_{i=1, \ldots, d}$ for $x \in C_{d}$.

2.1. Random vectors. Given $\left(X_{i, j}\right)_{1 \leqslant i \leqslant j \leqslant d}$ a family of independent random variables and $\left(e_{k, l}\right)_{1 \leqslant k \leqslant l \leqslant d} \subset C_{d}$ with $e_{k, k}=e_{k}, k=1, \ldots, d$, we define the random vector $X=\left(X_{1}, \ldots, X_{d}\right)$ by

$$
X_{i}:=\sum_{\substack{1 \leqslant k \leqslant l \leqslant d \\ e_{i} \preceq e_{k, l}}} X_{k, l}, \quad i=1, \ldots, d .
$$

In other words, we have

$$
\begin{aligned}
X & =\sum_{i=1}^{d} e_{i} X_{i}=\sum_{i=1}^{d} e_{i} \sum_{\substack{1 \leqslant k \leqslant l \leqslant d \\
e_{i} \preceq e_{k, l}}} X_{k, l} \\
& =\sum_{1 \leqslant k \leqslant l \leqslant d} X_{k, l} \sum_{\substack{1 \leqslant i \leqslant d \\
e_{i} \preceq e_{k, l}}} e_{i}=\sum_{1 \leqslant k \leqslant l \leqslant d} X_{k, l} e_{k, l},
\end{aligned}
$$

which implies

$$
E\left[X_{i}\right]=\sum_{\substack{1 \leqslant k \leqslant l \leqslant d \\ e_{i} \preceq e_{k, l}}} E\left[X_{k, l}\right], \quad i=1, \ldots, d
$$

and

$$
\operatorname{Cov}\left(X_{i}, X_{j}\right)=\sum_{\substack{1 \leqslant k \leqslant l \leqslant d \\ e_{i} \preceq e_{k, l}, e_{j} \preceq e_{k, l}}} \sigma_{k, l}^{2}, \quad 1 \leqslant i \leqslant j \leqslant d,
$$

where $\sigma_{k, l}^{2}:=\operatorname{Var}\left[X_{k, l}\right], 1 \leqslant k \leqslant l \leqslant d$.

EXAMPLE 2.1. If we take $d=5$, the subset $\left(e_{k, l}\right)_{1 \leqslant k \leqslant l \leqslant 5}$ of $C_{5}$ given by

$$
\left\{\begin{array}{l}
e_{1,2}=(1,1,0,0,1), \\
e_{1,3}=(1,1,1,0,1), \\
e_{1,4}=(1,0,0,1,0), \\
e_{1,5}=(1,0,0,0,1), \\
e_{2,3}=(0,1,1,0,0), \\
e_{2,4}=(0,1,0,1,0), \\
e_{2,5}=(0,1,0,0,1), \\
e_{3,4}=(0,1,1,1,0), \\
e_{3,5}=(0,1,1,0,1), \\
e_{4,5}=(1,0,0,1,1)
\end{array}\right.
$$




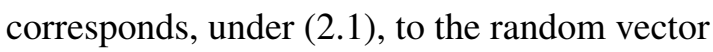

$$
\left\{\begin{array}{lll}
X_{1}=X_{1,1}+X_{1,2}+X_{1,3}+X_{1,4}+X_{1,5} & +X_{4,5} \\
X_{2}=X_{2,2}+X_{1,2}+X_{1,3} & +X_{2,3}+X_{2,4}+X_{2,5}+X_{3,4}+X_{3,5} \\
X_{3}=X_{3,3}+X_{1,3} & +X_{2,3}+X_{3,4}+X_{3,5} \\
X_{4}=X_{4,4}+X_{1,4} & +X_{2,4}+X_{3,4}+X_{4,5} \\
X_{5}=X_{5,5}+X_{1,2}+X_{1,3}+X_{1,5} & +X_{2,5}+X_{3,5}+X_{4,5} .
\end{array}\right.
$$

2.2. Binary tree structure. From now on, we work under the following Hypothesis $(\mathrm{H})$ that builds a tree on the set $\left(e_{k, l}\right)_{1 \leqslant k<l \leqslant d}$. Note that not all random vectors admit a tree-based representation according to Hypothesis $(\mathrm{H})$, see Examples 3.4, 3.5 and Counterexample 4.1 below.

(H) The family $\left(e_{k, l}\right)_{1 \leqslant k \leqslant l \leqslant d} \subset\{0,1\}^{d}$ forms an ordered binary tree for the partial order $\preceq$, in which every node $e_{k, l}, k<l$, has exactly two children $e_{k, l} \backslash\{k\}$ and $e_{k, l} \backslash\{l\}$.

We note that the tree $\left(e_{k, l}\right)_{1 \leqslant k \leqslant l \leqslant d}$ has size $d(d+1) / 2$ and height at most $d$.

The random vector $([2.3)$ of Example 2.1 satisfies Hypothesis $(\mathrm{H})$ with the following tree structure:

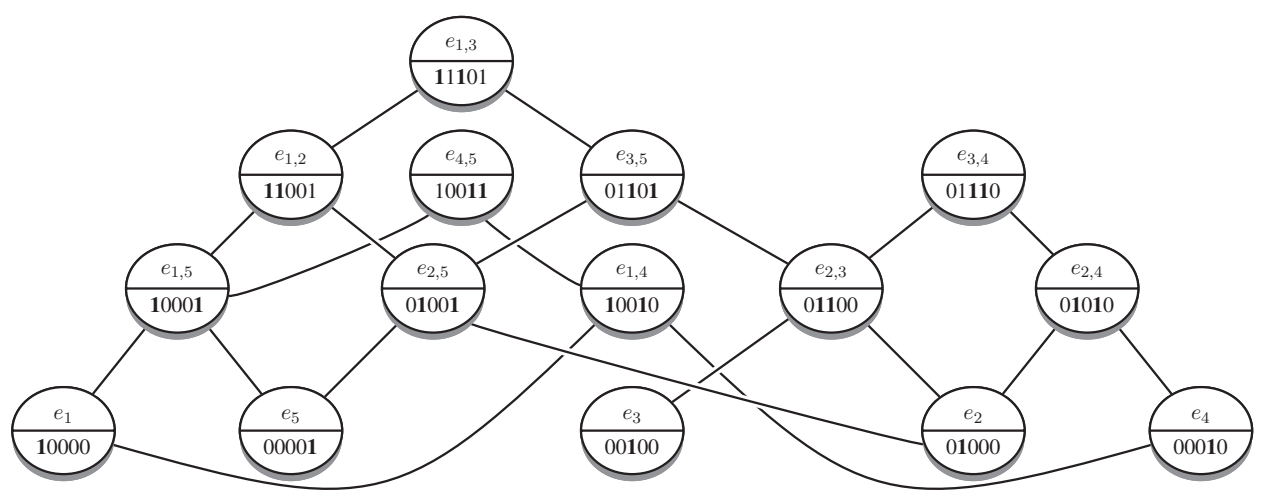

LEMMA 2.1. Under Hypothesis $(\mathrm{H})$ we have the equivalence

$$
e_{i, j} \preceq e_{k, l} \Longleftrightarrow\left(e_{i} \preceq e_{k, l} \text { and } e_{j} \preceq e_{k, l}\right)
$$

for all $1 \leqslant i \leqslant j \leqslant d$ and $1 \leqslant k \leqslant l \leqslant d$.

Pro of. (i) Assume that $e_{i, j} \preceq e_{k, l}$. Since both children $e_{i, j} \backslash\{i\}$ and $e_{i, j} \backslash\{j\}$ of $e_{i, j}$ satisfy $e_{i, j} \backslash\{i\} \prec e_{i, j}$ and $e_{i, j} \backslash\{j\} \prec e_{i, j}$, we have $e_{i} \preceq e_{i, j}$ and $e_{j} \preceq e_{i, j}$, which implies $e_{i} \preceq e_{k, l}$ and $e_{j} \preceq e_{k, l}$ since $e_{i, j} \preceq e_{k, l}$.

(ii) Assume that $e_{i} \preceq e_{k, l}$ and $e_{j} \preceq e_{k, l}$. We work by decreasing induction on the height of nodes in the tree. If $e_{k, l}=e_{k}$ is a leaf, i.e. $k=l$, then $\left(e_{i} \preceq e_{k, l}\right.$ and $\left.e_{j} \preceq e_{k, l}\right)$ implies $i=j=k=l$, hence $e_{i, j}=e_{i}=e_{k}=e_{k, l}$. Next, assuming that the conclusion holds for all nodes of height at least $h \geqslant 2$, consider a node $e_{k, l}$ of 
height $h-1$, with $k \neq l$. If $\left(e_{i} \preceq e_{k, l}\right.$ and $\left.e_{j} \preceq e_{k, l}\right)$ and $\{i, j\} \neq\{k, l\}$, we must have either $i \neq l$ and $j \neq l$, or $i \neq k$ and $j \neq k$. In the first case, $\left(e_{i} \preceq e_{k, l}\right.$ and $\left.e_{j} \preceq e_{k, l}\right)$ implies $e_{i} \preceq e_{k, l} \backslash\{l\}$ and $e_{j} \preceq e_{k, l} \backslash\{l\}$, where $e_{k, l} \backslash\{l\}$ has height $h$, hence $e_{i} \preceq e_{k, l} \backslash\{l\} \preceq e_{k, l}$ and $e_{j} \preceq e_{k, l} \backslash\{l\} \preceq e_{k, l}$ by the induction hypothesis. The conclusion is similar in the second case, by replacing $l$ with $k$.

Based on Lemma 2.1 , for all $1 \leqslant i \leqslant j \leqslant d$ we can now rewrite (2.2) as the sum

$$
\operatorname{Cov}\left(X_{i}, X_{j}\right)=\sum_{\substack{1 \leqslant k \leqslant l \leqslant d \\ e_{i, j} \preceq e_{k, l}}} \sigma_{k, l}^{2}, \quad 1 \leqslant i \leqslant j \leqslant d,
$$

over all couples $(k, l)$ with $e_{i, j} \preceq e_{k, l}$. In other words, $\left(\operatorname{Cov}\left(X_{i}, X_{j}\right)\right)_{1 \leqslant i \leqslant j \leqslant d}$ is the Möbius transform of $\left(\sigma_{k, l}^{2}\right)_{1 \leqslant k \leqslant l \leqslant d}$ on the partially ordered set $\left(\left(e_{k, l}\right)_{1 \leqslant k \leqslant l \leqslant d}, \preceq\right)$, cf. [13] or Section 2.5 of [12] for details.

\section{MÖBIUS INVERSION}

By Möbius inversion (cf. Proposition 2.6.3 of [12]), we can recover the coefficients $\left(\sigma_{k, l}^{2}\right)_{1 \leqslant k \leqslant l \leqslant d}$ in (2.2) using the covariances $\left(\operatorname{Cov}\left(X_{i}, X_{j}\right)\right)_{1 \leqslant i \leqslant j \leqslant d}$ as the sum

$$
\sigma_{k, l}^{2}=\sum_{\substack{1 \leqslant i \leqslant j \leqslant d \\ e_{k, l} \preceq e_{i, j}}} \mu\left(e_{i, j}, e_{k, l}\right) \operatorname{Cov}\left(X_{i}, X_{j}\right), \quad 1 \leqslant k \leqslant l \leqslant d,
$$

over all couples $(i, j)$ such that $e_{k, l} \preceq e_{i, j}, 1 \leqslant i \leqslant j \leqslant d$, where $\mu(x, y)$ is the Möbius function defined recursively by $\mu(x, x):=1$ and

$$
\mu(x, y):=-\sum_{y \prec z \preceq x} \mu(x, z), \quad x, y \in\{0,1\}^{d},
$$

cf. Proposition 2.6.1 of [12].

PROPOSITION 3.1.The Möbius function $\mu(x, y)$ on the tree $\left(\left(e_{k, l}\right)_{1 \leqslant k \leqslant l \leqslant d}, \preceq\right)$ is given by

$$
\left\{\begin{array}{l}
\mu\left(e_{k, l}, e_{k, l}\right)=1, \\
\mu\left(e_{k, l}, e_{k, l} \backslash\{k\}\right)=-1, \\
\mu\left(e_{k, l}, e_{k, l} \backslash\{l\}\right)=-1, \\
\mu\left(e_{k, l}, e_{k, l} \backslash\{k, l\}\right)=1, \quad 1 \leqslant k \leqslant l \leqslant d,
\end{array}\right.
$$

with $\mu\left(e_{k, l}, e_{i, j}\right)=0$ in all other cases. 
Pr o of. Given $e_{k, l} \in\{0,1\}^{d}$, we clearly have

$$
\mu\left(e_{k, l}, e_{k, l}\right)=1, \quad \mu\left(e_{k, l}, e_{k, l} \backslash\{k\}\right)=-1, \quad \text { and } \quad \mu\left(e_{k, l}, e_{k, l} \backslash\{l\}\right)=-1 .
$$

Next, since the two children $e_{k, l} \backslash\{k\}$ and $e_{k, l} \backslash\{l\}$ of $e_{k, l}$ have themselves a unique common child $e_{k, l} \backslash\{k, l\}$, (B.2) yields $\mu\left(e_{k, l}, e_{k, l} \backslash\{k, l\}\right)=1$.

The next graph, in which $y \notin\{k, l\}$, summarizes the result of Proposition B.J.

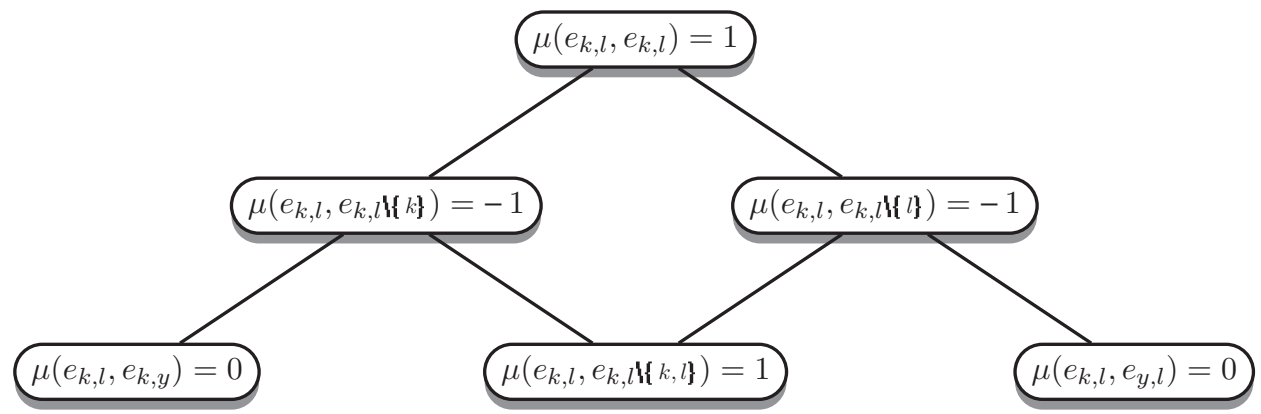

Using formula (B.]), we can now solve ([2.2) for $\left(\sigma_{k, l}^{2}\right)_{1 \leqslant k \leqslant l \leqslant d}$ starting from $\left(\operatorname{Cov}\left(X_{i}, X_{j}\right)\right)_{1 \leqslant i \leqslant j \leqslant d}$. However, not all these covariance matrices may lead to a positive solution $\left(\sigma_{k, l}^{2}\right)_{1 \leqslant k \leqslant l \leqslant d}$, meaning that not all random vectors admit a representation of the form (2.]), see Example 3.4 below.

EXAMPLE 3.1 (Comonotonic vectors). The comonotonic vector $\left(X_{k, l}, X_{k, l}\right.$, $\left.\ldots, X_{k, l}\right)$ can be represented by using a binary tree with a single node $e_{k, l}=$ $111 \ldots 111$ and letting $\sigma_{i, j}^{2}=0$ for $(i, j) \neq(k, l)$, since $\operatorname{Cov}\left(X_{i}, X_{j}\right)=\sigma_{k, l}^{2}$ for all $(i, j)$.

EXAmPle 3.2 (Pairwise dependence). The binary tree is reduced to the $d$ leaves $e_{1}, \ldots, e_{d}$, and to their parents $(d-1) d / 2$,

$$
e_{k, l}=(0, \ldots, \underset{\substack{\uparrow \\ k}}{0,1}, 0, \ldots, \underset{\substack{\uparrow \\ l}}{1}, 0, \ldots, 0), \quad 1 \leqslant k \leqslant l \leqslant d,
$$

as in the following example with $d=4$ :

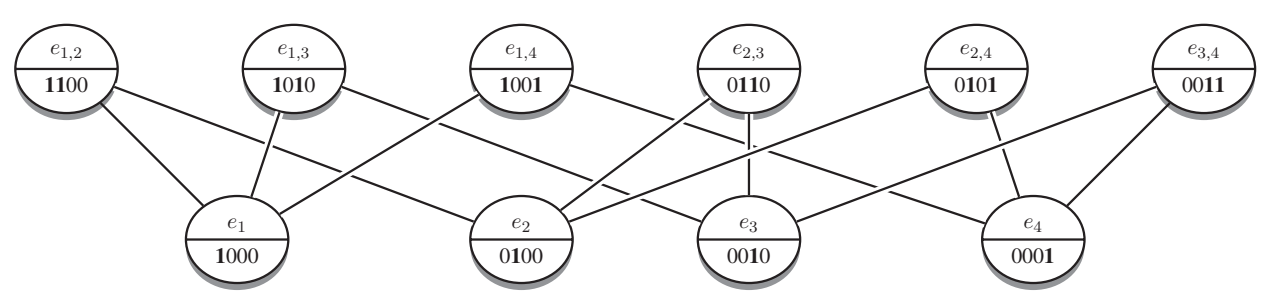


Here, the vector $\left(X_{i}\right)_{i=1, \ldots, d}$ is given by

$$
\left\{\begin{array}{lll}
X_{1}=X_{1,1}+X_{1,2}+X_{1,3}+X_{1,4} & & \\
X_{2}=X_{2,2}+X_{1,2} & +X_{2,3}+X_{2,4} & \\
X_{3}=X_{3,3}+X_{1,3} & +X_{2,3} & +X_{3,4} \\
X_{4}=X_{4,4}+X_{1,4} & +X_{2,4} & +X_{3,4},
\end{array}\right.
$$

and for any $d \geqslant 1$, by (2.2) we have

$$
\operatorname{Cov}\left(X_{i}, X_{j}\right)=\sigma_{i, j}^{2}, \quad 1 \leqslant i<j \leqslant d,
$$

and

$$
\operatorname{Var}\left[X_{i}\right]=\sum_{j=1}^{i-1} \sigma_{j, i}^{2}+\sum_{j=i}^{d} \sigma_{i, j}^{2}, \quad i=1, \ldots, d .
$$

Here, the inversion of (3.5) by the Möbius transform (3.D) reads

$$
\sigma_{k, k}^{2}=\operatorname{Var}\left[X_{k}\right]-\sum_{l=1, l \neq k}^{d} \operatorname{Cov}\left(X_{k}, X_{l}\right), \quad k=1, \ldots, d .
$$

EXAMPLE 3.3 (Recombining trees). In dimension $d=3$, the only available tree structure in addition to the pairwise dependence of Example 3.2 is the recombining (or binomial) full tree

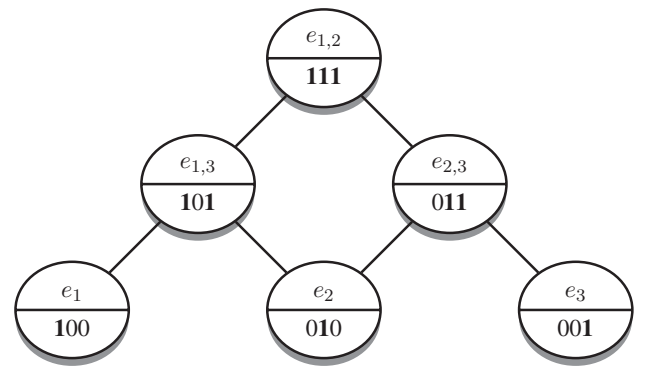

which is associated with the random vector

$$
\left\{\begin{array}{l}
X_{1}=X_{1,1}+X_{1,3}+X_{1,2} \\
X_{2}=X_{2,2}+X_{2,3}+X_{1,2} \\
X_{3}=X_{3,3}+X_{1,3}+X_{2,3}+X_{1,2},
\end{array}\right.
$$

with the inversion formula (B.D) written as

$$
\left\{\begin{array}{l}
\sigma_{1,1}^{2}=\operatorname{Cov}\left(X_{1}, X_{1}\right)-\operatorname{Cov}\left(X_{1}, X_{3}\right) \\
\sigma_{2,2}^{2}=\operatorname{Cov}\left(X_{2}, X_{2}\right)-\operatorname{Cov}\left(X_{1}, X_{3}\right)-\operatorname{Cov}\left(X_{2}, X_{3}\right)+\operatorname{Cov}\left(X_{1}, X_{2}\right) \\
\sigma_{3,3}^{2}=\operatorname{Cov}\left(X_{3}, X_{3}\right)-\operatorname{Cov}\left(X_{2}, X_{3}\right) \\
\sigma_{1,3}^{2}=\operatorname{Cov}\left(X_{1}, X_{3}\right)-\operatorname{Cov}\left(X_{1}, X_{2}\right) \\
\sigma_{2,3}^{2}=\operatorname{Cov}\left(X_{2}, X_{3}\right)-\operatorname{Cov}\left(X_{1}, X_{2}\right) \\
\sigma_{1,2}^{2}=\operatorname{Cov}\left(X_{1}, X_{2}\right)
\end{array}\right.
$$


EXAMPLE 3.4 (Multivariate Gaussian vectors). If $\left(X_{i, j}\right)_{1 \leqslant i \leqslant j \leqslant d}$ is a family of independent Gaussian random variables, then $X=\left(X_{1}, \ldots, X_{d}\right)$ in (Z.]) is a multivariate Gaussian vector with matrix $\left(\operatorname{Cov}\left(U_{i}, U_{j}\right)\right)_{1 \leqslant i \leqslant j \leqslant d}$ of nonnegative covariances given by (2.4). However, not all Gaussian vectors can fit into a treebased structure under Hypothesis $(\mathrm{H})$ above. For example, when $d=3$, consider the multivariate Gaussian vector

$$
\left\{\begin{array}{l}
X_{1}=X_{1,1}+X_{1,3}+X_{1,2}+Z \\
X_{2}=X_{2,2}+X_{2,3}+X_{1,2}+Z \\
X_{3}=X_{3,3}+X_{1,3}+X_{2,3}+X_{1,2}
\end{array}\right.
$$

where $\left(X_{k, l}\right)_{1 \leqslant k \leqslant l \leqslant d}$ are standard normal random variables and $Z$ is an independent Gaussian random variable with variance four. Here, $\left(X_{1}, X_{2}, X_{3}\right)$ has the (positive definite) covariance matrix

$$
\left(\begin{array}{lll}
\operatorname{Cov}\left(X_{1}, X_{1}\right) & \operatorname{Cov}\left(X_{1}, X_{2}\right) & \operatorname{Cov}\left(X_{1}, X_{3}\right) \\
\operatorname{Cov}\left(X_{1}, X_{2}\right) & \operatorname{Cov}\left(X_{2}, X_{2}\right) & \operatorname{Cov}\left(X_{2}, X_{3}\right) \\
\operatorname{Cov}\left(X_{1}, X_{3}\right) & \operatorname{Cov}\left(X_{2}, X_{3}\right) & \operatorname{Cov}\left(X_{3}, X_{3}\right)
\end{array}\right)=\left(\begin{array}{ccc}
7 & 5 & 2 \\
5 & 7 & 2 \\
2 & 2 & 4
\end{array}\right)
$$

in which case (B.6) cannot yield a nonnegative solution $\left(\sigma_{k, l}^{2}\right)_{1 \leqslant k \leqslant l \leqslant 3}$, e.g. when $(k, l)=(1,3)$. In this case, the multivariate Gaussian vector $\left(X_{1}, X_{2}, X_{3}\right)$ given by (B.7) admits no binary tree-based representation as the inversion formula (B.6) is based on a full tree.

EXAMPLE 3.5. As in Example 3.4 above, binomial, Poisson and gamma random vectors having a given matrix of nonnegative covariances can be constructed on a binary tree, provided that (B.J) admits a nonnegative solution $\left(\sigma_{k, l}^{2}\right)_{1 \leqslant k \leqslant l \leqslant 3}$ since their marginals are characterized by their variance parameters and they are stable by summation. However, in this case the construction may not be unique, depending on the chosen binary tree structure, as their joint distribution is not characterized by their covariance matrices.

EXAMPLE 3.6. The particular dependence structure considered in [ 7$]$ for Poisson random vectors corresponds to the binary tree built on the $d(d-1) / 2$ nodes

$$
e_{i, j}=(1, \ldots, 1, \underset{\substack{i \\ i}}{1}, 0, \ldots, 0, \underset{\substack{\uparrow \\ j}}{1}, 0, \ldots, 0), \quad 1 \leqslant i<j \leqslant d,
$$

and on the $d$ leaves $e_{1}, \ldots, e_{d}$.

\section{POISSON RANDOM VECTORS}

In this section we provide a characterization of the supermodular ordering of Poisson random vectors, based on their covariance matrices in Theorem 4.1. This extends the results of [ $[$ ] (cf. Example 3.6 above) to more general dependence structures. 
Recall that any $d$-dimensional infinitely divisible Poisson random vector $X=$ $\left(X_{1}, \ldots, X_{d}\right)$ is defined by its characteristic function

$$
E\left[e^{i\langle\bar{t}, X\rangle}\right]=\exp \left(\int_{\mathbb{R}^{d}}\left(e^{i\langle\bar{t}, x\rangle}-1\right) \mu(d x)\right)
$$

where $\bar{t}=\left(t_{1}, \ldots, t_{d}\right) \in \mathbb{R}^{d},\langle\cdot, \cdot\rangle$ denotes the scalar product in $\mathbb{R}^{d}$, and the Lévy measure

$$
\mu(d x):=\sum_{y \in\{0,1\}^{d}} a_{y} \delta_{y}(d x)
$$

is supported on $C_{d}=\{0,1\}^{d}$. Here $\delta_{y}$ denotes the Dirac measure at the point $y \in\{0,1\}^{d}$, and $\left(a_{y}\right)_{y \in C_{d}}$ is a family of nonnegative coefficients with $a_{(0, \ldots, 0)}=0$.

Equivalently, $X=\left(X_{1}, \ldots, X_{d}\right)$ can be represented as

$$
X_{i}=\sum_{y \in\{0,1\}^{d}} \mathbf{1}_{\{i \in y\}} Z_{y}=\sum_{y \in C_{d}, e_{i} \preceq y} Z_{y}, \quad i=1, \ldots, d,
$$

where $\left(Z_{y}\right)_{y \in C_{d} \backslash\{0\}}$ is a family of $2^{d}-1$ independent Poisson random variables with respective intensities $\left(a_{y}\right)_{y \in C_{d} \backslash\{0\}}$, cf. also Example 4.3 of [4] and Theorem 3 of [6].

To characterize the ordering of Poisson random vectors based on the data of their covariance matrices which contain only $d(d+1) / 2$ components, we consider Lévy measures of the form

$$
\mu(d x)=\sum_{1 \leqslant k \leqslant l \leqslant d} a_{k, l} \delta_{e_{k, l}}(d x),
$$

on $\{0,1\}^{d}$, where $a_{k, l} \in \mathbb{R}_{+}, 1 \leqslant k \leqslant l \leqslant d$. In this case, (4.]) rewrites as

$$
X_{i}=\sum_{\substack{1 \leqslant k \leqslant l \leqslant d \\ e_{i} \preceq e_{k, l}}} X_{k, l}
$$

where $\left(X_{k, l}\right)_{1 \leqslant k \leqslant l \leqslant d}$ is a family of independent Poisson random variables whose respective intensities $\left(a_{i, j}\right)_{1 \leqslant i \leqslant j \leqslant d}$ satisfy $\operatorname{Var}\left[X_{k, l}\right]=E\left[X_{k, l}\right]=a_{k, l}, 1 \leqslant k \leqslant$ $l \leqslant d$.

In the remaining of this section we assume that the family $\left(e_{k, l}\right)_{1 \leqslant k \leqslant l \leqslant d} \subset$ $\{0,1\}^{d}$ forms a binary tree according to Hypothesis $(\mathrm{H})$. In this case, the Möbius inversion formula (B.T) shows that

$$
a_{k, l}=\sum_{\substack{1 \leqslant i \leqslant j \leqslant d \\ e_{k, l} \preceq e_{i, j}}} \mu\left(e_{i, j}, e_{k, l}\right) \operatorname{Cov}\left(X_{i}, X_{j}\right), \quad 1 \leqslant k \leqslant l \leqslant d .
$$


4.1. Supermodular ordering of Poisson random vectors. Theorem th. below is a direct consequence of the following Lemma 4.1] which yields the decomposition

$$
\begin{aligned}
\mu(d x)= & \sum_{i=1}^{d} \operatorname{Var}\left[X_{i}\right] \delta_{e_{i}}(d x) \\
& +\sum_{1 \leqslant i<j \leqslant d} \operatorname{Cov}\left(X_{i}, X_{j}\right)\left(\delta_{e_{i, j}}+\delta_{e_{i, j} \backslash\{i, j\}}-\delta_{e_{i, j} \backslash\{i\}}-\delta_{e_{i, j} \backslash\{j\}}\right)(d x)
\end{aligned}
$$

of a Lévy measure $\mu(d x)$ of the form (4.2) under Hypothesis $(\mathrm{H})$.

LemMa 4.1. Let $\left(X_{1}, \ldots, X_{d}\right)$ be an infinitely divisible Poisson random vector written as in (4.3) under Hypothesis $(\mathrm{H})$, with Lévy measure $\mu(d x)$ on $C_{d}$. Then we have

$$
\begin{aligned}
& \text { (4.5) } \int_{\mathbb{R}^{d}} \phi(x) \mu(d x)=\sum_{i=1}^{d} E\left[X_{i}\right] \phi\left(e_{i}\right) \\
& +\sum_{1 \leqslant i<j \leqslant d} \operatorname{Cov}\left(X_{i}, X_{j}\right)\left(\phi\left(e_{i, j}\right)+\phi\left(e_{i, j} \backslash\{i, j\}\right)-\phi\left(e_{i, j} \backslash\{i\}\right)-\phi\left(e_{i, j} \backslash\{j\}\right)\right)
\end{aligned}
$$

for any function $\phi:\{0,1\}^{d} \rightarrow \mathbb{R}$ such that $\phi(0)=0$.

P r o o f. By the Möbius inversion formula (B.D) we have

$$
\begin{aligned}
& \int_{\mathbb{R}^{d}} \phi(x) \mu(d x)=\sum_{1 \leqslant k \leqslant l \leqslant d} a_{k, l} \phi\left(e_{k, l}\right) \\
= & \sum_{1 \leqslant k \leqslant l \leqslant d} \phi\left(e_{k, l}\right) \sum_{\substack{1 \leqslant i \leqslant j \leqslant d \\
e_{k, l} \preceq e_{i, j}}} \mu\left(e_{i, j}, e_{k, l}\right) \operatorname{Cov}\left(X_{i}, X_{j}\right) \\
= & \sum_{i=1}^{d} \operatorname{Cov}\left(X_{i}, X_{i}\right) \sum_{\substack{1 \leqslant k \leqslant d \\
e_{k} \preceq e_{i}}} \mu\left(e_{i}, e_{k}\right) \phi\left(e_{k}\right) \\
& +\sum_{1 \leqslant i<j \leqslant d} \operatorname{Cov}\left(X_{i}, X_{j}\right) \sum_{\substack{1 \leqslant k<l \leqslant d \\
e_{k}, l}} \mu\left(e_{i, j}\right. \\
= & \left.\sum_{i=1}^{d} E\left[X_{i}\right] \phi\left(e_{i}\right) e_{k, l}\right) \phi\left(e_{k, l}\right) \\
& +\sum_{1 \leqslant i<j \leqslant d} \operatorname{Cov}\left(X_{i}, X_{j}\right)\left(\phi\left(e_{i, j}\right)+\phi\left(e_{i, j} \backslash\{i, j\}\right)-\phi\left(e_{i, j} \backslash\{i\}\right)-\phi\left(e_{i, j} \backslash\{j\}\right)\right),
\end{aligned}
$$

where we used (B.3a), (3.3b) and the fact that $e_{k} \preceq e_{i}$ if and only if $k=i$. 
EXAMPLE 4.1. For $d=4$, the tree structure

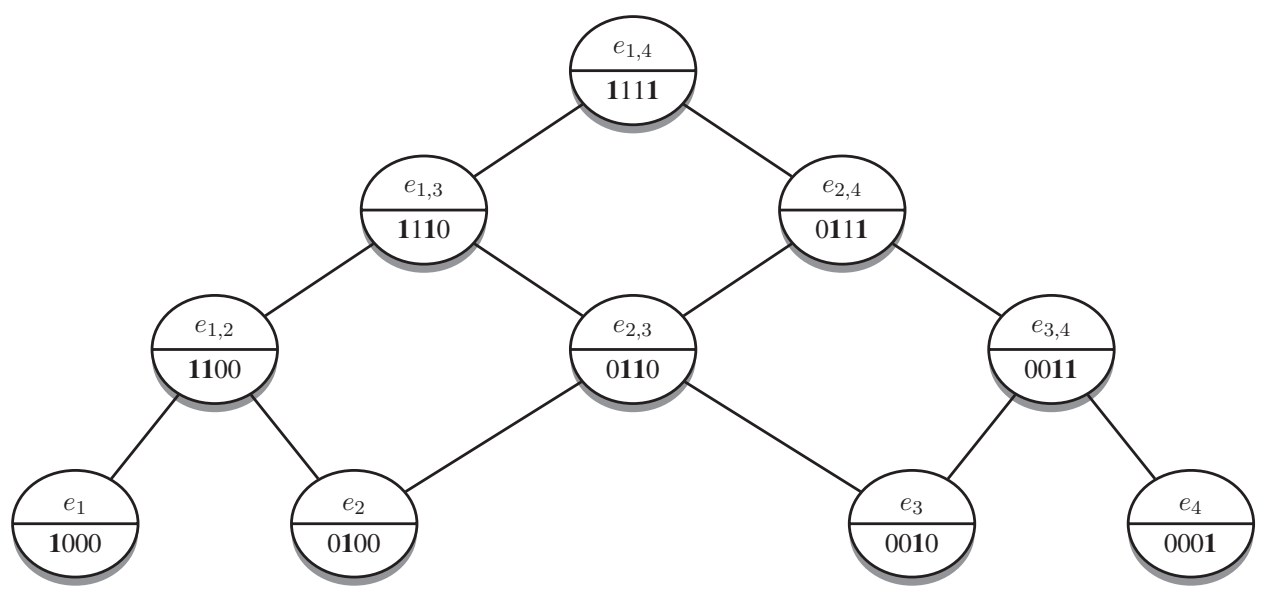

is satisfied by the random vector

$$
\left\{\begin{array}{l}
X_{1}=X_{1,1}+X_{1,2}+X_{1,3}+X_{1,4} \\
X_{2}=X_{2,2}+X_{1,2}+X_{1,3}+X_{1,4}+X_{2,3}+X_{2,4} \\
X_{3}=X_{3,3}+X_{1,3}+X_{1,4}+X_{2,3}+X_{2,4}+X_{3,4} \\
X_{4}=X_{4,4}+X_{1,4}+X_{2,4}+X_{3,4}
\end{array}\right.
$$

and relation (4.5) reads

$$
\begin{aligned}
& \int_{\mathbb{R}^{d}} \phi(x) \mu(d x)=a_{1,4} \phi(1,1,1,1)+a_{1,3} \phi(1,1,1,0)+a_{2,4} \phi(0,1,1,1) \\
& +a_{1,2} \phi(1,1,0,0)+a_{2,3} \phi(0,1,1,0)+a_{3,4} \phi(0,0,1,1) \\
& +a_{1,1} \phi(1,0,0,0)+a_{2,2} \phi(0,1,0,0)+a_{3,3} \phi(0,0,1,0)+a_{4,4} \phi(0,0,0,1) \\
= & E\left[X_{1}\right] \phi(1,0,0,0)+E\left[X_{2}\right] \phi(0,1,0,0) \\
& +E\left[X_{3}\right] \phi(0,0,1,0)+E\left[X_{4}\right] \phi(0,0,0,1) \\
& +\operatorname{Cov}\left(X_{1}, X_{2}\right)(\phi(1,1,0,0)+\phi(0,0,0,0)-\phi(1,0,0,0)-\phi(0,1,0,0)) \\
& +\operatorname{Cov}\left(X_{1}, X_{3}\right)(\phi(1,1,1,0)+\phi(0,1,0,0)-\phi(1,1,0,0)-\phi(0,1,1,0)) \\
& +\operatorname{Cov}\left(X_{1}, X_{4}\right)(\phi(1,1,1,1)+\phi(0,1,1,0)-\phi(1,1,1,0)-\phi(0,1,1,1)) \\
& +\operatorname{Cov}\left(X_{2}, X_{3}\right)(\phi(0,1,1,0)+\phi(0,0,0,0)-\phi(0,1,0,0)-\phi(0,0,1,0)) \\
+ & \operatorname{Cov}\left(X_{2}, X_{4}\right)(\phi(0,1,1,1)+\phi(0,0,1,0)-\phi(0,1,1,0)-\phi(0,0,1,1)) \\
+ & \operatorname{Cov}\left(X_{3}, X_{4}\right)(\phi(0,0,1,1)+\phi(0,0,0,0)-\phi(0,0,1,0)-\phi(0,0,0,1)) .
\end{aligned}
$$

Consider now two Poisson random vectors $X$ and $Y$ whose respective Lévy measures $\mu$ and $\nu$ are represented as in (4.2), i.e.,

$$
\mu(d x)=\sum_{1 \leqslant i \leqslant j \leqslant d} a_{i, j} \delta_{e_{i, j}}(d x) \quad \text { and } \quad \nu(d x)=\sum_{1 \leqslant i \leqslant j \leqslant d} b_{i, j} \delta_{e_{i, j}}(d x) .
$$


If $X_{i}$ has the same distribution as $Y_{i}$ for all $i=1, \ldots, d$, then $E\left[X_{i}\right]=E\left[Y_{i}\right]$, $i=1, \ldots, d$, and Lemma 4.$]$ l shows that

$$
\begin{aligned}
& \int_{\mathbb{R}^{d}} \phi(y) \nu(d y)-\int_{\mathbb{R}^{d}} \phi(x) \mu(d x) \\
& =\sum_{1 \leqslant i<j \leqslant d}\left(\operatorname{Cov}\left(Y_{i}, Y_{j}\right)-\operatorname{Cov}\left(X_{i}, X_{j}\right)\right)\left(\phi\left(e_{i, j}\right)+\phi\left(e_{i, j} \backslash\{i, j\}\right)\right. \\
& \left.\quad-\phi\left(e_{i, j} \backslash\{i\}\right)-\phi\left(e_{i, j} \backslash\{j\}\right)\right)
\end{aligned}
$$

under Hypothesis (H). Relation (4.6) implies in particular that the nonnegativity of the coefficients

$$
\operatorname{Cov}\left(Y_{i}, Y_{j}\right)-\operatorname{Cov}\left(X_{i}, X_{j}\right) \geqslant 0, \quad 1 \leqslant i<j \leqslant d,
$$

becomes a necessary and sufficient condition for the supermodular ordering of the Lévy measures $\mu$ and $\nu$.

The following Theorem 4.1] reformulates (4.7) as a necessary and sufficient condition for supermodular ordering of infinitely divisible Poisson random vectors, based on Theorem 4.5 of [I] , which allows one to carry over the notion of supermodularity from the setting of Lévy measures on the discrete cube $C_{d}=\{0,1\}^{d}$ to the setting of Poisson random variables.

THEOREM 4.1. Consider two Poisson random vectors $X$ and $Y$ both represented as in (4.3) under Hypothesis (H). Then the conditions

$$
E\left[X_{i}\right]=E\left[Y_{i}\right], \quad 1 \leqslant i \leqslant d,
$$

and

$$
\operatorname{Cov}\left(X_{i}, X_{j}\right) \leqslant \operatorname{Cov}\left(Y_{i}, Y_{j}\right), \quad 1 \leqslant i<j \leqslant d,
$$

are necessary and sufficient for the supermodular ordering $X \leqslant_{\mathrm{sm}} Y$.

Pr o of. It is well known (cf. e.g. Theorem 3.9.5 of [II]) that for any couple $(X, Y)$ of $d$-dimensional random vectors, the condition $X \leqslant_{\mathrm{sm}} Y$ implies (4.8) and (4.9), therefore it suffices to show sufficiency. For this, by Theorem 4.5 in [U] it suffices to show that we have

$$
\int_{\mathbb{R}^{d}} \phi(x) \mu(d x) \leqslant \int_{\mathbb{R}^{d}} \phi(y) \nu(d y)
$$

for all supermodular functions $\phi: \mathbb{R}^{d} \rightarrow \mathbb{R}$, where $\mu(d x)$ and $\nu(d y)$ denote the Lévy measures of $X$ and $Y$, respectively. By Lemma 4.1 we have the identity (4.6) under condition (4.9), which allows us to conclude that (4.10) holds for all supermodular functions $\phi$. 
Next, we consider a situation where Hypothesis $(\mathrm{H})$ is not satisfied and the equivalence of Theorem 4.1$]$ does not hold.

Counterexample 4.1. If we take $d=4$, the tree

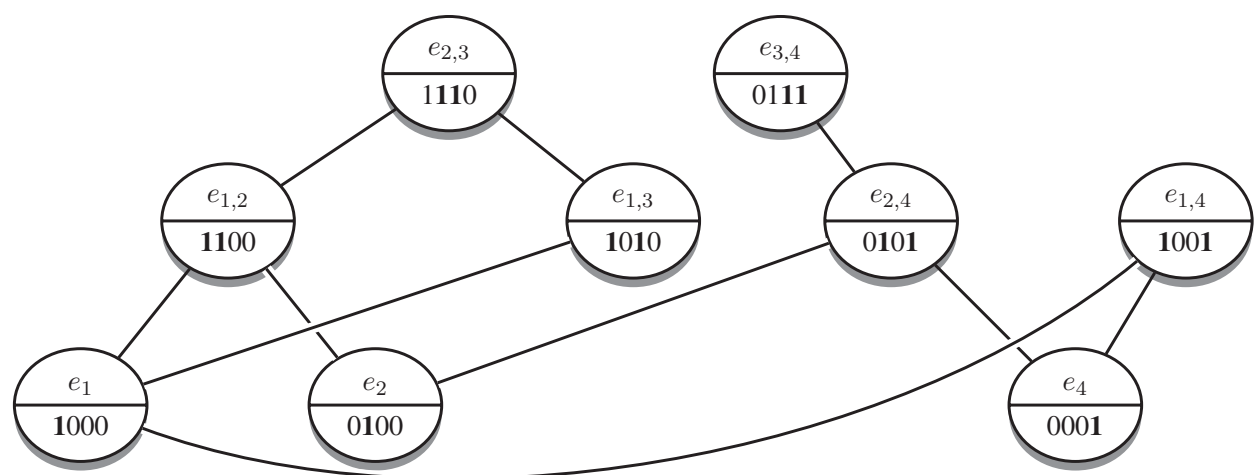

does not satisfy Hypothesis $(\mathrm{H})$, and for its corresponding random vector

$$
\begin{cases}X_{1}=X_{1,1}+X_{1,2}+X_{1,3}+X_{1,4} & +X_{2,3} \\ X_{2}=X_{2,2}+X_{1,2} & +X_{2,3}+X_{2,4}+X_{3,4} \\ X_{3}= & +X_{2,3}+X_{3,4} \\ X_{4}=X_{4,4}+X_{1,3}+X_{2,4}+X_{3,4}\end{cases}
$$

relation (4.11) reads

$$
\begin{aligned}
& \int_{\mathbb{R}^{d}} \phi(x) \mu(d x)=a_{2,3} \phi(1,1,1,0)+a_{3,4} \phi(0,1,1,1) \\
& +a_{1,2} \phi(1,1,0,0)+a_{1,3} \phi(1,0,1,0)+a_{2,4} \phi(0,1,0,1)+a_{1,4} \phi(1,0,0,1) \\
& +a_{1,1} \phi(1,0,0,0)+a_{2,2} \phi(0,1,0,0)+a_{4,4} \phi(0,0,0,1) \\
= & E\left[X_{1}\right] \phi(1,0,0,0)+E\left[X_{2}\right] \phi(0,1,0,0) \\
& +E\left[X_{3}\right] \phi(0,0,1,0)+E\left[X_{4}\right] \phi(0,0,0,1) \\
& +\operatorname{Cov}\left(X_{1}, X_{2}\right)(\phi(1,1,0,0)+\phi(0,0,0,0)-\phi(1,0,0,0)-\phi(0,1,0,0)) \\
& +\operatorname{Cov}\left(X_{1}, X_{3}\right)(\phi(1,0,1,0)+\phi(0,0,0,0)-\phi(1,0,0,0)-\phi(0,0,1,0)) \\
& +\operatorname{Cov}\left(X_{1}, X_{4}\right)(\phi(1,0,0,1)+\phi(0,0,0,0)-\phi(1,0,0,0)-\phi(0,0,0,1)) \\
& +\operatorname{Cov}\left(X_{2}, X_{3}\right)(\phi(1,1,1,0)+\phi(1,0,0,0)-\phi(1,1,0,0)-\phi(1,0,1,0)) \\
& +\operatorname{Cov}\left(X_{2}, X_{4}\right)(\phi(0,1,0,1)+\phi(0,0,0,0)-\phi(0,1,0,0)-\phi(0,0,0,1)) \\
& +\operatorname{Cov}\left(X_{3}, X_{4}\right)(\phi(0,1,1,1)+\phi(0,0,0,0)-\phi(0,1,0,1)-\phi(0,0,1,0)) \\
& -\operatorname{Cov}\left(X_{3}, X_{4}\right)(\phi(1,1,1,0)+\phi(1,0,0,0)-\phi(1,0,1,0)-\phi(1,1,0,0)) .
\end{aligned}
$$

In this case, the conclusion of Theorem 4.11 cannot hold for vectors of the form (4.II) as the sum of the above two terms in factor of $\operatorname{Cov}\left(X_{3}, X_{4}\right)$ can become negative, e.g. for the supermodular function $\phi\left(x_{1}, x_{2}, x_{3}, x_{4}\right)=x_{1} x_{2} x_{3}$ on the unit cube. 
The next proposition replaces the equality of means in (4.8) with an inequality, and is obtained as in Proposition 4.3 of [ [ $]$ ] by extending Theorem 4.5 of [i]] to nondecreasing supermodular functions $\phi$ on $\mathbb{R}^{d}$ satisfying $\phi(0)=0$, using the same approximation as in Lemma 4.4 therein.

Proposition 4.1. Consider two Poisson random vectors $X$ and $Y$ both represented as in (4.3) under Hypothesis $(\mathrm{H})$, and assume that

$$
E\left[X_{i}\right] \leqslant E\left[Y_{i}\right], \quad 1 \leqslant i \leqslant d,
$$

and

$$
\operatorname{Cov}\left(X_{i}, X_{j}\right) \leqslant \operatorname{Cov}\left(Y_{i}, Y_{j}\right), \quad 1 \leqslant i<j \leqslant d .
$$

Then we have

$$
E[\Phi(X)] \leqslant E[\Phi(Y)]
$$

for all nondecreasing supermodular functions $\Phi: \mathbb{R}^{d} \rightarrow \mathbb{R}$.

4.2. Convex ordering. The next result is a remark on the convex ordering of Poisson random vectors represented as in (4.3).

Proposition 4.2. Consider two Poisson random vectors $X$ and $Y$ both represented as in (4.3) under Hypothesis $(\mathrm{H})$. Then we have $X \leqslant_{\mathrm{cx}} Y$ if and only if $X$ and $Y$ have the same distribution.

Pro of. We assume that $X \leqslant_{\mathrm{cx}} Y$, i.e., we have $E[\Phi(X)] \leqslant E[\Phi(Y)]$ for all convex functions $\Phi: \mathbb{R}^{d} \rightarrow \mathbb{R}$. Clearly, this implies $E\left[X_{k}\right]=E\left[Y_{k}\right], k=1, \ldots, d$, and by the same argument as in part (b) of the proof of Theorem 4.5 in [I] we also have $\mu \leqslant_{\mathrm{cx}} \nu$. Assume now that $\operatorname{Cov}\left(Y_{k}, Y_{l}\right)>\operatorname{Cov}\left(X_{k}, X_{l}\right)$ for some $1 \leqslant k<$ $l \leqslant d$. The function

$$
\left(x_{1}, \ldots, x_{d}\right) \mapsto \phi_{k, l}\left(x_{1}, \ldots, x_{d}\right):=\max \left(0, x_{l}-x_{k}-\sum_{a \notin e_{k, l}} x_{a}\right)
$$

is convex on $\mathbb{R}^{d}$ and satisfies $\phi_{k, l}\left(e_{i, j}\right)=1$ when $e_{i, j}$ is a (non-strict) descendant of $e_{k, l} \backslash\{k\}$ that contains $l$, and $\phi_{k, l}\left(e_{i, j}\right)=0$ in all other cases. This shows that

$$
\phi_{k, l}\left(e_{k, l}\right)+\phi_{k, l}\left(e_{k, l} \backslash\{k, l\}\right)-\phi_{k, l}\left(e_{k, l} \backslash\{k\}\right)-\phi_{k, l}\left(e_{k, l} \backslash\{l\}\right)=-1,
$$

and

$$
\phi_{k, l}\left(e_{i, j}\right)+\phi_{k, l}\left(e_{i, j} \backslash\{i, j\}\right)-\phi_{k, l}\left(e_{i, j} \backslash\{i\}\right)-\phi_{k, l}\left(e_{i, j} \backslash\{j\}\right)=0
$$

when $(i, j) \neq(k, l)$. Therefore, since $\operatorname{Cov}\left(Y_{k}, Y_{l}\right)>\operatorname{Cov}\left(X_{k}, X_{l}\right)$, Lemma 4.] shows that 


$$
\begin{aligned}
\int_{\mathbb{R}^{d}} \phi(y) & \nu(d y)-\int_{\mathbb{R}^{d}} \phi(x) \mu(d x) \\
= & \sum_{1 \leqslant i<j \leqslant d}\left(\operatorname{Cov}\left(Y_{i}, Y_{j}\right)-\operatorname{Cov}\left(X_{i}, X_{j}\right)\right)\left(\phi\left(e_{i, j}\right)+\phi\left(e_{i, j} \backslash\{i, j\}\right)\right. \\
& \left.-\phi\left(e_{i, j} \backslash\{i\}\right)-\phi\left(e_{i, j} \backslash\{j\}\right)\right) \\
= & \left(\operatorname{Cov}\left(Y_{k}, Y_{l}\right)-\operatorname{Cov}\left(X_{k}, X_{l}\right)\right)\left(\phi\left(e_{k, l}\right)+\phi\left(e_{k, l} \backslash\{k, l\}\right)\right. \\
& \left.-\phi\left(e_{k, l} \backslash\{k\}\right)-\phi\left(e_{k, l} \backslash\{l\}\right)\right) \\
< & 0,
\end{aligned}
$$

which contradicts the fact that $\mu \leqslant{ }_{\mathrm{cx}} \nu$, hence $\operatorname{Cov}\left(Y_{k}, Y_{l}\right) \leqslant \operatorname{Cov}\left(X_{k}, X_{l}\right)$. If $\operatorname{Cov}\left(Y_{k}, Y_{l}\right)<\operatorname{Cov}\left(X_{k}, X_{l}\right)$, we can proceed similarly with the convex function

$$
\left(x_{1}, \ldots, x_{d}\right) \mapsto-\phi_{k, l}\left(x_{1}, \ldots, x_{d}\right),
$$

and conclude that $\operatorname{Cov}\left(Y_{k}, Y_{l}\right)=\operatorname{Cov}\left(X_{k}, X_{l}\right)$ for all $1 \leqslant k \leqslant l \leqslant d$, hence by (4.4) the vectors $X$ and $Y$ have the same distribution.

\section{BINOMIAL RANDOM VECTORS}

In this section we provide a characterization of the supermodular ordering of binomial random vectors, based on their covariance matrices, cf. Theorem 5.1 .

Consider $\left(Z_{1}, \ldots, Z_{n}\right)$ independent Bernoulli random variables with parameter $p \in[0,1]$ and $\left(A\left(e_{k, l}\right)\right)_{1 \leqslant k \leqslant l \leqslant d}$ a partition of $\{1, \ldots, n\}$. Let $\left(X_{k, l}\right)_{1 \leqslant k \leqslant l \leqslant d}=$ $\left(X_{A\left(e_{k, l}\right)}\right)_{1 \leqslant k \leqslant l \leqslant d}$ denote the family of independent binomial random variables given by

$$
X_{k, l}=X_{A\left(e_{k, l}\right)}:=\sum_{i \in A\left(e_{k, l}\right)} Z_{i}, \quad 1 \leqslant k \leqslant l \leqslant d,
$$

with

$$
E\left[X_{A\left(e_{k, l}\right)}\right]=p\left|A\left(e_{k, l}\right)\right|, \quad 1 \leqslant k \leqslant l \leqslant d,
$$

where $\left|A\left(e_{k, l}\right)\right|$ denotes the cardinality of $A\left(e_{k, l}\right)$, and

$$
\sigma_{k, l}^{2}=\operatorname{Var}\left[X_{A\left(e_{k, l}\right)}\right]=p q\left|A\left(e_{k, l}\right)\right|, \quad 1 \leqslant k \leqslant l \leqslant d, q:=1-p .
$$

Let now

$$
A_{i}:=\bigcup_{\substack{1 \leqslant k \leqslant l \leqslant d \\ e_{i} \preceq e_{k, l}}} A\left(e_{k, l}\right), \quad i=1, \ldots, d,
$$

and consider the vector $\left(X_{1}, \ldots, X_{d}\right)=\left(X_{A_{1}}, \ldots, X_{A_{d}}\right)$ of binomial random variables defined by

$$
X_{i}=X_{A_{i}}:=\sum_{k \in A_{i}} Z_{k}=\sum_{\substack{1 \leqslant k \leqslant l \leqslant d \\ e_{i} \preceq e_{k, l}}} X_{A\left(e_{k, l}\right)}, \quad i=1, \ldots, d .
$$


In general, we have

$$
E\left[X_{A_{i}}\right]=p \sum_{\substack{1 \leqslant k \leqslant l \leqslant d \\ e_{i} \preceq e_{k, l}}}\left|A\left(e_{k, l}\right)\right|, \quad i=1, \ldots, d,
$$

and

$$
\operatorname{Cov}\left(X_{A_{i}}, X_{A_{j}}\right)=p q \sum_{\substack{1 \leqslant k \leqslant l \leqslant d \\ e_{i, j} \preceq e_{k, l}}}\left|A\left(e_{k, l}\right)\right|, \quad 1 \leqslant i \leqslant j \leqslant d .
$$

Assuming that the family $\left(e_{k, l}\right)_{1 \leqslant k \leqslant l \leqslant d} \subset C_{d}$ forms a binary tree according to Hypothesis (H), the Möbius inversion formula (B.J) shows that we have

$$
p q\left|A\left(e_{k, l}\right)\right|=\sum_{\substack{1 \leqslant k \leqslant d \\ e_{k, l} \preceq e_{i, j}}} \mu\left(e_{i, j}, e_{k, l}\right) \operatorname{Cov}\left(X_{A_{i}}, X_{A_{j}}\right), \quad 1 \leqslant k \leqslant l \leqslant d .
$$

The following is the main result of this section.

Theorem 5.1. Consider $\left(X_{A_{1}}, \ldots, X_{A_{d}}\right)$ and $\left(X_{B_{1}}, \ldots, X_{B_{d}}\right)$ two binomial random vectors represented as in (5.]) under Hypothesis $(\mathrm{H})$. Then the conditions

$$
E\left[X_{A_{i}}\right]=E\left[X_{B_{i}}\right], \quad 1 \leqslant i \leqslant d,
$$

and

$$
\operatorname{Cov}\left(X_{A_{i}}, X_{A_{j}}\right) \leqslant \operatorname{Cov}\left(X_{B_{i}}, X_{B_{j}}\right), \quad 1 \leqslant i<j \leqslant d,
$$

are necessary and sufficient for the supermodular ordering

$$
\left(X_{A_{1}}, \ldots, X_{A_{d}}\right) \leqslant_{\mathrm{sm}}\left(X_{B_{1}}, \ldots, X_{B_{d}}\right) .
$$

Pro of. By Theorem 3.9.5 of [U]], it suffices to show sufficiency. Using induction, it is also sufficient to consider the case where

$$
\operatorname{Cov}\left(X_{B_{k}}, X_{B_{l}}\right)=\operatorname{Cov}\left(X_{A_{k}}, X_{A_{l}}\right)+p q
$$

for some given $1 \leqslant k<l \leqslant d$, and

$$
\operatorname{Cov}\left(X_{B_{i}}, X_{B_{j}}\right)=\operatorname{Cov}\left(X_{A_{i}}, X_{A_{j}}\right), \quad 1 \leqslant i \leqslant j \leqslant d,(i, j) \neq(k, l) .
$$

By the Möbius inversion formula (5.2), there is a unique way (up to a permutation of $\{1, \ldots, n\})$ to choose $\left(A\left(e_{i, j}\right)\right)_{1 \leqslant i \leqslant j \leqslant d}$ and $\left(B\left(e_{i, j}\right)\right)_{1 \leqslant i \leqslant j \leqslant d}$ satisfying (5.5) and (5.6), respectively. In this case, (B. I) shows that

$$
p q\left|B\left(e_{i, j}\right)\right|=\sum_{\substack{1 \leqslant x \leqslant y \leqslant d \\ e_{i, j} \preceq e_{x, y}}} \mu\left(e_{x, y}, e_{i, j}\right) \operatorname{Cov}\left(X_{B_{x}}, X_{B_{y}}\right)
$$




$$
\begin{aligned}
& =p q \mathbf{1}_{\left\{e_{i, j} \preceq e_{k, l}\right\}} \mu\left(e_{k, l}, e_{i, j}\right)+\sum_{\substack{1 \leqslant x \leqslant y \leqslant d \\
e_{i, j} \preceq e_{x, y}}} \mu\left(e_{x, y}, e_{i, j}\right) \operatorname{Cov}\left(X_{A_{x}}, X_{A_{y}}\right) \\
& =p q \mathbf{1}_{\left\{e_{i, j} \preceq e_{k, l}\right\}} \mu\left(e_{k, l}, e_{i, j}\right)+p q\left|A\left(e_{i, j}\right)\right|, \quad 1 \leqslant i \leqslant j \leqslant d,
\end{aligned}
$$

i.e.,

$$
\left|B\left(e_{i, j}\right)\right|=\mathbf{1}_{\left\{e_{i, j} \preceq e_{k, l}\right\}} \mu\left(e_{k, l}, e_{i, j}\right)+\left|A\left(e_{i, j}\right)\right|, \quad 1 \leqslant i \leqslant j \leqslant d .
$$

Given the children $e_{k, l} \backslash\{k\}, e_{k, l} \backslash\{l\} \in\{0,1\}^{d}$ and grandchild $e_{k, l} \backslash\{k, l\}$ of $e_{k, l} \in$ $\{0,1\}^{d}$, by (B.3a), (B.3b) and (5.7), we have

$$
\left\{\begin{array}{l}
\left|B\left(e_{k, l}\right)\right|=\left|A\left(e_{k, l}\right)\right|+1, \\
\left|B\left(e_{k, l} \backslash\{k\}\right)\right|=\left|A\left(e_{k, l} \backslash\{k\}\right)\right|-1, \\
\left|B\left(e_{k, l} \backslash\{l\}\right)\right|=\left|A\left(e_{k, l} \backslash\{l\}\right)\right|-1, \\
\left|B\left(e_{k, l} \backslash\{k, l\}\right)\right|=\left|A\left(e_{k, l} \backslash\{k, l\}\right)\right|+1,
\end{array}\right.
$$

with $\left|B\left(e_{i, j}\right)\right|=\left|A\left(e_{i, j}\right)\right|$, since $\mu\left(e_{k, l}, e_{i, j}\right)=0$, in all other cases. We choose to realize the above as

$$
\left\{\begin{array}{l}
A\left(e_{k, l}\right)=B\left(e_{k, l}\right) \backslash\{k\}, \\
B\left(e_{k, l} \backslash\{k\}\right)=A\left(e_{k, l} \backslash\{k\}\right) \backslash\{k\}, \\
B\left(e_{k, l} \backslash\{l\}\right)=A\left(e_{k, l} \backslash\{l\}\right) \backslash\{l\}, \\
A\left(e_{k, l} \backslash\{k, l\}\right)=B\left(e_{k, l} \backslash\{k, l\}\right) \backslash\{l\}
\end{array}\right.
$$

for some given $1 \leqslant k<l \leqslant d$, with $k, l \notin B\left(e_{i, j}\right)=A\left(e_{i, j}\right)$ in all other cases. Noting that

$$
l \in B\left(e_{k, l} \backslash\{k, l\}\right), \quad k \in A\left(e_{k, l} \backslash\{k\}\right), \quad l \in A\left(e_{k, l} \backslash\{l\}\right),
$$

and

$$
\begin{gathered}
B\left(e_{k, l} \backslash\{k, l\}\right) \cap B_{k}=\emptyset, \quad B\left(e_{k, l} \backslash\{k, l\}\right) \cap B_{l}=\emptyset, \\
A\left(e_{k, l} \backslash\{k\}\right) \cap A_{k}=\emptyset, \quad A\left(e_{k, l} \backslash\{l\}\right) \cap A_{l}=\emptyset,
\end{gathered}
$$

we find that

$$
l \notin B_{k}, \quad l \notin B_{l}, \quad k \notin A_{k}, \quad l \notin A_{l} .
$$


Hence, using the symmetric difference operator $A \backslash B:=A \cap B^{c}$, for $i=1, \ldots, d$ we have

$$
A_{i}=\left\{\begin{array}{cc}
\left(B_{k} \backslash B\left(e_{k, l}\right) \backslash B\left(e_{k, l} \backslash\{k, l\}\right)\right) \cup A\left(e_{k, l}\right) \cup\{l\}, & i=k, \\
\left(B_{i} \backslash B\left(e_{k, l}\right) \backslash B\left(e_{k, l} \backslash\{k, l\}\right)\right) \cup A\left(e_{k, l}\right) \cup\{k\} \cup A\left(e_{k, l} \backslash\{k, l\}\right) \cup\{l\}, & i \notin\{k, l\}, \\
\left(B_{l} \backslash B\left(e_{k, l}\right) \backslash B\left(e_{k, l} \backslash\{k, l\}\right)\right) \cup A\left(e_{k, l}\right) \cup\{k\}, & i=l,
\end{array}\right.
$$

and

(5.11)

$$
B_{i}= \begin{cases}\left(B_{k} \backslash B\left(e_{k, l}\right) \backslash B\left(e_{k, l} \backslash\{k, l\}\right)\right) \cup B\left(e_{k, l}\right), & i=k, \\ \left(B_{i} \backslash B\left(e_{k, l}\right) \backslash B\left(e_{k, l} \backslash\{k, l\}\right)\right) \cup B\left(e_{k, l}\right) \cup B\left(e_{k, l} \backslash\{k, l\}\right), & i \notin\{k, l\}, \\ \left(B_{l} \backslash B\left(e_{k, l}\right) \backslash B\left(e_{k, l} \backslash\{k, l\}\right)\right) \cup B\left(e_{k, l}\right), & i=l .\end{cases}
$$

In other words, by (5.9) we can write

$$
\left\{\begin{array}{l}
X_{B\left(e_{k, l}\right)}=X_{A\left(e_{k, l}\right)}+U, \\
X_{A\left(e_{k, l} \backslash\{k\}\right)}=X_{B\left(e_{k, l} \backslash\{k\}\right)}+U, \\
X_{A\left(e_{k, l} \backslash\{l\}\right)}=X_{B\left(e_{k, l} \backslash\{l\}\right)}+V, \\
X_{B\left(e_{k, l} \backslash\{k, l\}\right)}=X_{A\left(e_{k, l} \backslash\{k, l\}\right)}+V,
\end{array}\right.
$$

where $U, V \in\left\{Z_{1}, \ldots, Z_{n}\right\}$ are two independent Bernoulli random variables, while we have $X_{B\left(e_{i, j}\right)}=X_{A\left(e_{i, j}\right)}$ in all other cases, and from (5.TU) and (5.TI) we get (5.13)

$$
X_{A_{i}}= \begin{cases}X_{B_{k} \backslash B\left(e_{k, l}\right) \backslash B\left(e_{k, l} \backslash\{k, l\}\right)}+X_{A\left(e_{k, l}\right)}+V, & i=k, \\ X_{B_{i} \backslash B\left(e_{k, l}\right) \backslash B\left(e_{k, l} \backslash\{k, l\}\right)}+X_{A\left(e_{k, l}\right)}+U+X_{A\left(e_{k, l} \backslash\{k, l\}\right)}+V, & i \notin\{k, l\}, \\ X_{B_{l} \backslash B\left(e_{k, l}\right) \backslash B\left(e_{k, l} \backslash\{k, l\}\right)}+X_{A\left(e_{k, l}\right)}+U, & i=l,\end{cases}
$$

and

$$
X_{B_{i}}= \begin{cases}X_{B_{k} \backslash B\left(e_{k, l}\right) \backslash B\left(e_{k, l} \backslash\{k, l\}\right)}+X_{B\left(e_{k, l}\right)}, & i=k, \\ X_{B_{i} \backslash B\left(e_{k, l}\right) \backslash B\left(e_{k, l} \backslash\{k, l\}\right)}+X_{B\left(e_{k, l}\right)}+X_{B\left(e_{k, l} \backslash\{k, l\}\right)}, & i \notin\{k, l\}, \\ X_{B_{l} \backslash B\left(e_{k, l}\right) \backslash B\left(e_{k, l} \backslash\{k, l\}\right)}+X_{B\left(e_{k, l}\right)}, & i=l .\end{cases}
$$

Now, for any supermodular function $\phi: \mathbb{R}^{d} \rightarrow \mathbb{R}$ we have, using formulas (5.14) and (5.12), 


$$
\begin{aligned}
& E\left[\phi\left(\left(X_{B_{i}}\right)_{1 \leqslant i \leqslant d}\right)\right] \\
= & E\left[\phi\left(\left(X_{B_{i} \backslash B\left(e_{k, l}\right) \backslash B\left(e_{k, l} \backslash\{k, l\}\right)}+X_{B\left(e_{k, l}\right)}+X_{B\left(e_{k, l} \backslash\{k, l\}\right)} \mathbf{1}_{\{i \notin\{k, l\}\}}\right)_{1 \leqslant i \leqslant d}\right)\right] \\
= & E\left[\phi \left(\left(X_{B_{i} \backslash B\left(e_{k, l}\right) \backslash B\left(e_{k, l} \backslash\{k, l\}\right)}+X_{A\left(e_{k, l}\right)}+U\right.\right.\right. \\
& \left.\left.\left.+\left(X_{A\left(e_{k, l} \backslash\{k, l\}\right)}+V\right) \mathbf{1}_{\{i \notin\{k, l\}\}}\right)_{1 \leqslant i \leqslant d}\right)\right] \\
\geqslant & E\left[\phi \left(\left(X_{B_{i} \backslash B\left(e_{k, l}\right) \backslash B\left(e_{k, l} \backslash\{k, l\}\right)}+X_{A\left(e_{k, l}\right)}+U \mathbf{1}_{\{i \neq k\}}\right.\right.\right. \\
& \left.\left.\left.+X_{A\left(e_{k, l} \backslash\{k, l\}\right)} \mathbf{1}_{\{i \notin\{k, l\}\}}+V \mathbf{1}_{\{i \neq l\}}\right)_{1 \leqslant i \leqslant d}\right)\right] \\
= & E\left[\phi\left(\left(X_{A_{i}}\right)_{1 \leqslant i \leqslant d}\right)\right],
\end{aligned}
$$

where we used (5.13) for the last equality. As for the inequality above, it follows from

$$
\begin{aligned}
E[\phi(U, U & +V, \ldots, U+V, U)] \\
= & p^{2} \phi(1,2, \ldots, 2,1)+q^{2} \phi(0,0, \ldots, 0,0)+p q \phi(1,1, \ldots, 1,1) \\
& +p q \phi(0,1, \ldots, 1,0) \\
\geqslant & p^{2} \phi(1,2, \ldots, 2,1)+q^{2} \phi(0,0, \ldots, 0,0)+p q \phi(1,1, \ldots, 1,0) \\
& +p q \phi(0,1, \ldots, 1,1) \\
= & E[\phi(U, U+V, \ldots, U+V, V)]
\end{aligned}
$$

for all supermodular functions $\phi: \mathbb{R}^{\left|e_{k, l}\right|} \rightarrow \mathbb{R}$, where $\left|e_{k, l}\right|$ denotes the cardinality of $e_{k, l}$ whose indices are arranged as $\{k, \ldots, l\}$ for convenience of notation, and we did not consider indices $j \notin e_{k, l}$, as $U$ and $V$ do not belong to $X_{j}$ in this case.

5.1. Multivariate Gaussian vectors. From the central limit theorem, Theorem 5.$]$ can be used to deal with centered multivariate Gaussian random vectors $\left(X_{1}, \ldots, X_{d}\right)$ and $\left(Y_{1}, \ldots, Y_{d}\right)$ represented as in Example 3.4 as

$$
X=\sum_{1 \leqslant k \leqslant l \leqslant d} X_{k, l} e_{k, l}, \quad Y=\sum_{1 \leqslant k \leqslant l \leqslant d} Y_{k, l} e_{k, l}
$$

where $\left(e_{k, l}\right)_{1 \leqslant k \leqslant l \leqslant d} \subset\{0,1\}^{d}$ satisfies Hypothesis (H). In this case we can apply the Möbius inversion (B.]) to determine the variance coefficients

$$
\left(\sigma_{k, l}^{2}\right)_{1 \leqslant k \leqslant l \leqslant d}=\left(\operatorname{Var}\left[X_{k, l}\right]\right)_{1 \leqslant k \leqslant l \leqslant d} \quad \text { and } \quad\left(\eta_{k, l}^{2}\right)_{1 \leqslant k \leqslant l \leqslant d}=\left(\operatorname{Var}\left[Y_{k, l}\right]\right)_{1 \leqslant k \leqslant l \leqslant d}
$$

in the decomposition (5.15). Those coefficients can be obtained as the respective limits of normalized variances $\left(\operatorname{Var}\left[X_{k, l}^{n}\right] / n\right)_{1 \leqslant k \leqslant l \leqslant d}$ and $\left(\operatorname{Var}\left[Y_{k, l}^{n}\right] / n\right)_{1 \leqslant k \leqslant l \leqslant d}$ of independent binomial random variables $\left(X_{k, l}^{n}\right)_{1 \leqslant k \leqslant l \leqslant d}$ and $\left(Y_{k, l}^{n}\right)_{1 \leqslant k \leqslant l \leqslant d}$. In this case, the sequences $\left(X_{1}^{n}, \ldots, X_{d}^{n}\right)_{n \geqslant 1}$ and $\left(Y_{1}^{n}, \ldots, Y_{d}^{n}\right)_{n \geqslant 1}$ of independent 
random vectors defined by

$$
X_{i}^{n}:=\frac{1}{\sqrt{n}} \sum_{\substack{1 \leqslant k \leqslant l \leqslant d \\ e_{i} \preceq e_{k, l}}}\left(X_{k, l}^{n}-E\left[X_{k, l}^{n}\right]\right)
$$

and

$$
Y_{i}^{n}:=\frac{1}{\sqrt{n}} \sum_{\substack{1 \leqslant k \leqslant l \leqslant d \\ e_{i} \preceq e_{k, l}}}\left(Y_{k, l}^{n}-E\left[Y_{k, l}^{n}\right]\right), \quad i=1, \ldots, d,
$$

converge in distribution to the multivariate Gaussian vectors $\left(X_{1}, \ldots, X_{d}\right)$ and $\left(Y_{1}, \ldots, Y_{d}\right)$, respectively. The condition $\operatorname{Cov}\left(X_{i}, X_{j}\right) \leqslant \operatorname{Cov}\left(Y_{i}, Y_{j}\right)$ shows that $\operatorname{Cov}\left(X_{i}^{n}, X_{j}^{n}\right) \leqslant \operatorname{Cov}\left(Y_{i}^{n}, Y_{j}^{n}\right)$ for $n$ sufficiently large, $1 \leqslant i<j \leqslant d$, so by Theorem 5.1 it becomes necessary and sufficient for $\left(X_{1}, \ldots, X_{d}\right) \leqslant \mathrm{sm}\left(Y_{1}, \ldots, Y_{d}\right)$ to hold. This is consistent with the general result proved for all multivariate Gaussian random vectors in [10], Theorem 4.2, cf. also Theorem 3.13.5 of [II]].

A similar limiting argument can be applied to recover Theorem H.d in the Poisson case from Theorem 5.1$]$ and the convergence in distribution of renormalized binomial random variables to Poisson random variables.

5.2. Sums of binomial, Gaussian and Poisson vectors. By Theorem 4.2 of [10] on Gaussian random vectors, Theorems [5.] and 4.1] above, and the fact that the supermodular ordering is closed under convolution, cf. Theorem 3.9.14-(C) of [II], we deduce that the supermodular ordering of a sum of independent binomial, Gaussian and Poisson vectors is implied by the componentwise ordering of their respective covariances. Proposition 4.1 admits an analog extension to sums of binomial, Gaussian and Poisson random vectors.

\section{REFERENCES}

[1] N. Bäuerle, A. Blatter, and A. Müller, Dependence properties and comparison results for Lévy processes, Math. Methods Oper. Res. 67 (1) (2008), pp. 161-186.

[2] M. Denuit, E. Frostig, and B. Levikson, Supermodular comparison of time-to-ruin random vectors, Methodol. Comput. Appl. Probab. 9 (1) (2007), pp. 41-54.

[3] E. Frostig, Ordering ruin probabilities for dependent claim streams, Insurance Math. Econom. 32 (1) (2003), pp. 93-114.

[4] E. Frostig and F. Pellerey, Supermodular comparison of dependence models and multivariate processes, with applications, in: Recent Advances in Probability and Statistics, Lect. Notes Semin. Interdiscip. Mat., Vol. 12, Potenza, 2015, pp 125-138.

[5] T. Hu, C. Xie, and L. Ruan, Dependence structures of multivariate Bernoulli random vectors, J. Multivariate Anal. 94 (1) (2005), pp. 172-195.

[6] K. Kawamura, The structure of multivariate Poisson distribution, Kodai Math. J. 2 (3) (1979), pp. 337-345.

[7] B. Kiz1ldemir and N. Privault, Supermodular ordering of Poisson arrays, Statist. Probab. Lett. 98 (2015), pp. 136-143.

[8] M. A. Meyer and B. Strulovici, The supermodular stochastic ordering, CEPR Discussion Paper DP9486, May 2013. http://www.cepr.org/pubs/dps/DP9486. 
[9] A. Müller, Stop-loss order for portfolios of dependent risks, Insurance Math. Econom. 21 (3) (1997), pp. 219-223.

[10] A. Müller and M. Scarsini, Some remarks on the supermodular order, J. Multivariate Anal. 73 (1) (2000), pp. 107-119.

[11] A. Müller and D. Stoyan, Comparison Methods for Stochastic Models and Risks, Wiley, Chichester 2002.

[12] G. Peccati and M. S. Taqqu, Wiener Chaos: Moments, Cumulants and Diagrams. A Survey with Computer Implementation. Supplementary Material Available Online, Springer, Milan 2011.

[13] G. Rota, On the foundations of combinatorial theory: I. Theory of Möbius functions, Z. Wahrsch. Verw. Gebiete 2 (1964), pp. 340-368.

Bünyamin Kızıldemir

Division of Mathematical Sciences

School of Physical and Mathematical Sciences

Nanyang Technological University

637371 Singapore

E-mail: bunyamin001@e.ntu.edu.sg
Nicolas Privault

Division of Mathematical Sciences School of Physical and Mathematical Sciences Nanyang Technological University 637371 Singapore E-mail:nprivault@ntu.edu.sg

Received on 21.11.2016;

revised version on 5.5.2017 